\title{
Comparação da Eficácia de Aparatos de Isolamento para Testes Comportamentais em Cyprinidae, Danio Rerio
}

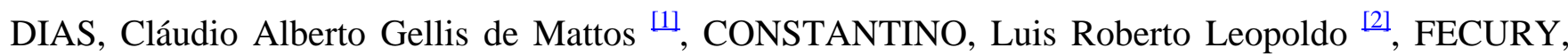
Amanda Alves ${ }^{[3]}$, OLIVEIRA, Euzébio de ${ }^{[4]}$, SANTOS, Bruno Rodrigues dos ${ }^{[5]}$, MANSUR, Bruno de Matos ${ }^{[6]}$, DENDASCK, Carla Viana ${ }^{[7]}$, JUNIOR, Amauri Gouveia ${ }^{[8]}$

DIAS, Cláudio Alberto Gellis de Mattos; et.al. Comparação da Eficácia de Aparatos de Isolamento para Testes Comportamentais em Cyprinidae, Danio Rerio. Revista Científica Multidisciplinar Núcleo do Conhecimento. Edição 09. Ano 02, Vol. 04. pp 133-139, Dezembro de 2017. ISSN:2448-0959

\section{RESUMO}

O Danio rerio é largamente utilizado como modelo experimental, pois possui uma manutenção fácil e barata e é um peixe ornamental muito bem adaptado à vida em aquários. Estudos conduzidos com peixes demonstram o efeito ansiogênico que ambientes brancos ocasionam quando sujeitos são colocados em aquários com uma metade branca e outra metade preta para testes de exploração. Estudos realizados com Zebrafish demonstram a preferência desta espécie pelo ambiente escuro e sua aversão ao ambiente claro. Esta nota científica tem o objetivo de comparar dois aparatos de isolamento em testes comportamentais utilizando o ciprinídeo Danio rerio, Zebrafish. Foram isolados, em cada aparato, 08 sujeitos, por sete dias, em aquários transparentes idênticos. Os sujeitos, um por vez, foram testados em um aquário teste branco/preto. O tempo de permanência em cada lado do aquário foi computado. Não há diferença estatisticamente significante entre eles $(\mathrm{F}(1,28)=0,000, \mathrm{p}=1,000)$. Ambos os aparatos demonstraram ser igualmente eficazes na manutenção do Zebrafish para testes comportamentais com variável luminosa.

Palavras-Chave: Aparato, Preferencia Claro/Escuro, Danio Rerio, Zebrafish.

\section{INTRODUÇÃO}

O Zebrafish é um pequeno peixe ósseo (aproximadamente 30 milímetros), da ordem dos Ciprinídeos, oriundo do delta do rio Ganges, Índia subcontinental (localização aproximada $22^{\circ} \mathrm{N}, 90^{\circ} \mathrm{E}$, próximo ao Trópico de Câncer). Apresenta hábitos diurnos (Spence, Gerlach et al., 2008) e originalmente vive em águas claras e limpas, sob pontes, em fossos ou lagos rasos que tenham vegetação abundante às suas margens. Observado desde a superfície até o fundo da coluna de água do seu habitat, comumente entre a 
vegetação e em plantações de arroz (Spence, Fatema et al., 2006). Seu crescimento, após a fecundação, ocorre de maneira rápida nos três primeiros meses desacelerando até os dezoito meses, em uma vida média de aproximadamente quarenta e dois meses (Spence, Gerlach et al., 2008).

O Danio rerio, é largamente utilizado como modelo experimental, pois possui uma manutenção fácil e barata e é um peixe ornamental muito bem adaptado à vida em aquários (Houart, 2001). A regularidade da postura de ovos pelas fêmeas, o número de embriões gerados, o desenvolvimento e o amadurecimento sexual rápidos são algumas de suas vantagens (Bilotta e Saszik, 2001).

Estudos conduzidos com peixes demonstram o efeito ansiogênico que ambientes brancos ocasionam quando sujeitos são colocados em aquários com uma metade branca e outra metade preta para testes de exploração (Gouveia Jr, Zampieri et al., 2005; Maximino, Brito et al., 2010).

Estudos realizados com Zebrafish demonstram a preferência desta espécie pelo ambiente escuro e sua aversão ao ambiente claro. Serra (1999) verificou a preferência do paulistinha por ambientes escuros em dois experimentos que utilizaram um aquário com uma metade branca e outra metade preta, separados por um anteparo removível. O primeiro foi realizado em dois dias consecutivos, com os mesmos animais, e consistia em colocá-los, um por vez, ou no lado branco ou no preto por trinta segundos, retirando depois o anteparo e deixando-os explorar por dez minutos. Em ambos os dias o tempo de permanência em cada lado do aquário foi registrado. O segundo experimento repetiu o primeiro, sendo que metade dos animais, inicialmente, foram postos no lado branco do aquário e a outra metade no lado preto, um por teste. $\mathrm{O}$ tempo medido foi o que o animal levava para passar do lado em que foi colocado para o lado oposto do aquário.

A preferência pelo lado escuro em aquário teste é bastante resistente a manipulações ambientais, não sendo afetada pelo enriquecimento ambiental do alojamento, isolamento do animal, formato aquário, entre outras variáveis (Maximino, Brito et al., 2009). Essa preferência apresenta um efeito de habituação espécie-dependente, não sendo relatada para o Danio rerio, mas sim no Carassius auratus (Brito, Maximino et al., 2007). Dias (2010) demonstrou a preferência do Danio rerio pelo lado escuro ao isolar grupos em ambientes com diferentes períodos de luminosidade. Ficou evidente também fator de arrastamento que o fotoperíodo ocasiona em grupos de Zebrafish, depois testá-los em diferentes períodos, em um aquário como o de Serra (1999).

\section{OBJETIVO}

Comparar a eficiência de dois aparatos de isolamento em testes comportamentais utilizando o ciprinídeo Danio rerio, Zebrafish.

\section{MATERIAL E MÉTODOS}

\section{ISOLAMENTOS}

Foram isolados, em cada aparato, 08 sujeitos, por sete dias, em dois diferentes apartos. Cada um desses aparatos teve sua luminosidade controlada por temporizadores (timers) ligados a lâmpadas internas, num ciclo com 12 horas de luminosidade por 12 horas de escuro total (denominado 12/12). 


\begin{abstract}
APARATOS
O aparato 1 foi construído com placas de MDF (aglomerado de alta densidade) de quinze milímetros, suas medidas são: 64,5 x 79,0 x 42,0 centímetros. É dividido em quatro compartimentos de 30,0 x 30,0 x 40,0 centímetros. Cada compartimento possui uma porta vedada duplamente com fita espuma autocolante de três milímetros, instaladas uma na beirada interna da porta e outra, na mesma posição, no aparato, o que garante a vedação luminosa completa do mesmo. Lâmpadas internas, em cada compartimento, ligadas a temporizadores (timers) garantem o controle do período luminoso. Orifícios dotados de ventoinhas (coolers) retiram o ar quente de cada compartimento, independentemente, através de orifícios que não permitem a entrada ou saída de luz. Abaixo dos quatro compartimentos há uma gaveta de $61,0 \mathrm{x}$ 12,0 x 40,0 centímetros com função de armazenamento de insumos. Na parte superior, o aparato possui um tampo de acrílico branco de cinco milímetros de espessura, 64,5 centímetros de comprimento e 42,0 centímetros de profundidade. Para aferição da temperatura, manutenção da aeração dos aquários de isolamento e alimentação sem abrir os compartimentos existe respectivamente termômetros com visor externo, bomba de ar e tubos com abertura externa.
\end{abstract}

O aparato 2 foi construído com placas de MDF (aglomerado de alta densidade) de seis milímetros. Suas medidas são: 60,0 x 30,0 x 40,0 centímetros (comprimento x profundidade x altura). Possui uma tampa com bordas internas, o que proporciona vedação luminosa completa ao mesmo, quando fechado. Uma lâmpada interna, ligada a um temporizador (timer) deverá controlar o fotoperíodo de cada um dos aparatos. Ventoinhas (coolers) retiram o ar quente através de orifícios não permitindo a entrada ou saída de luz. Para aferição da temperatura da água, manutenção da aeração dos aquários de isolamento e alimentação sem abrir os compartimentos (portanto sem afetar o fotoperíodo de cada aparato) foram instalados, respectivamente, termômetros com visor externo, bombas de ar e tubos com abertura externa (Figura 1). 


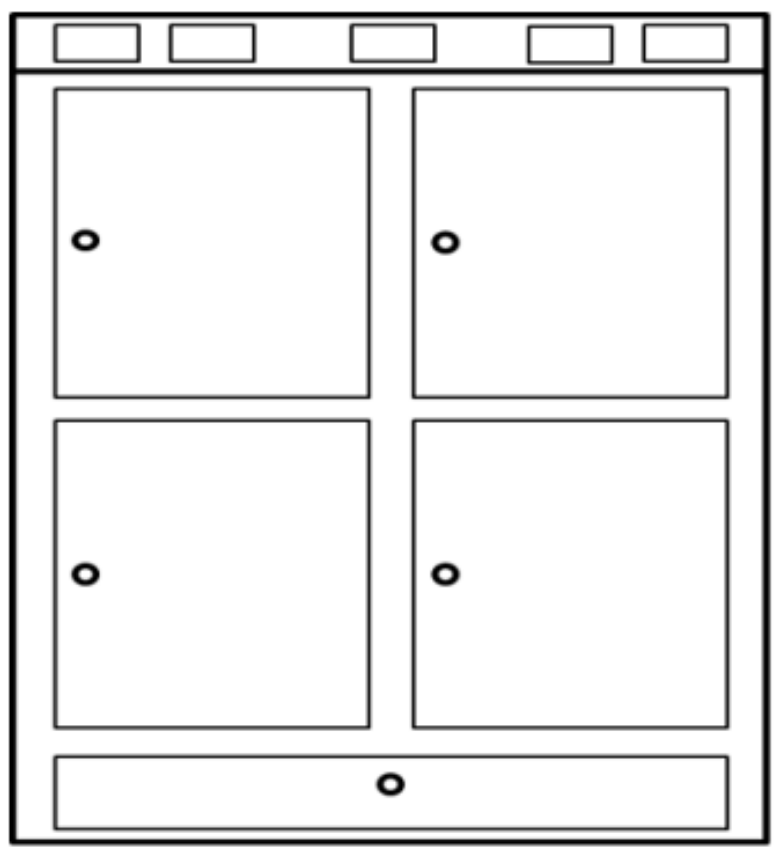

Aparato 1

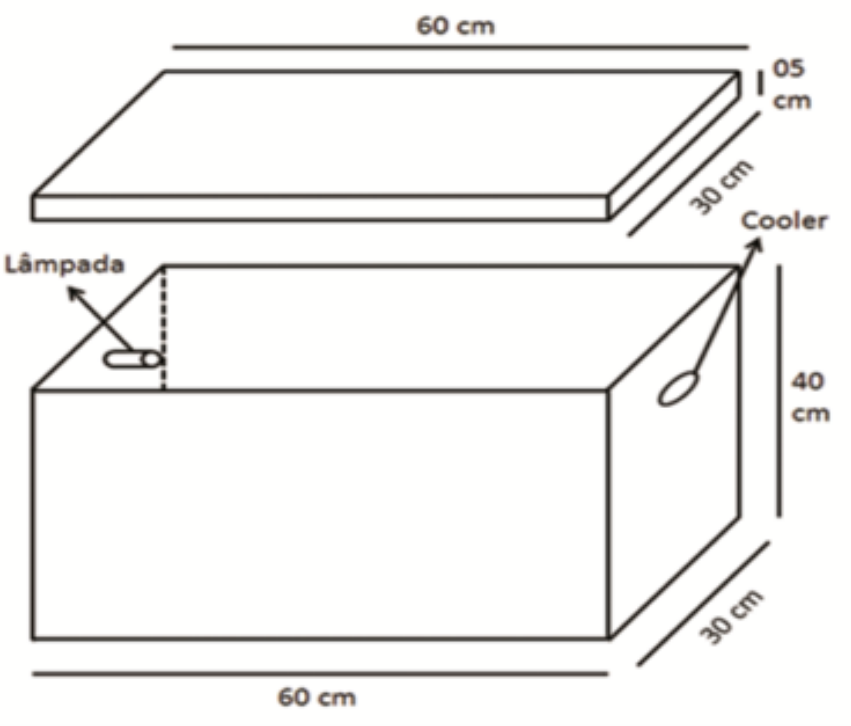

Aparato 2

Figura 1 - Esquemas simples mostrando a aparência externa dos aparatos 1 e 2.

\section{TESTE}

Os sujeitos, um por vez, foram testados em um aquário teste branco/preto $(45,0 \times 15,0 \times 10,0$ centímetros), composto por uma metade completamente preta e a outra completamente branca, com um espaço central de cinco centímetros, delimitado por duas comportas removíveis. Ocorreu um tempo de cinco minutos (300 segundos) para habituação no espaço delimitado pelas comportas. Após este tempo as comportas foram removidas e o sujeito pôde explorar o aquário por quinze minutos (900 segundos). $\mathrm{O}$ tempo de permanência em cada lado do aquário, a primeira latência (tempo que o sujeito leva para ir ao lado oposto à primeira escolha) e o número de cruzamentos foram computados.

\section{ESTATÍSTICA}

Os dados obtidos foram analisados estatisticamente por ANOVA de uma via com o programa BioStat 2009, com pós-teste Tukey.

\section{RESULTADOS}

Os resultados obtidos, após análise (ANOVA de duas vias,e pós-teste Tukey) comparando os aparatos, não apontou diferença estatisticamente significante entre eles $(F(1,28)=0,000, p=1,000)$, sem diferenças entre aparatos $(\mathrm{F}(1,28)=0,000, \mathrm{p}=1,000)$ e sem interação $\mathrm{F}(1,28)=1,620, \mathrm{p}=0,214$. Os dados demonstram a mesma eficiência entre os aparatos (Figura 2). 


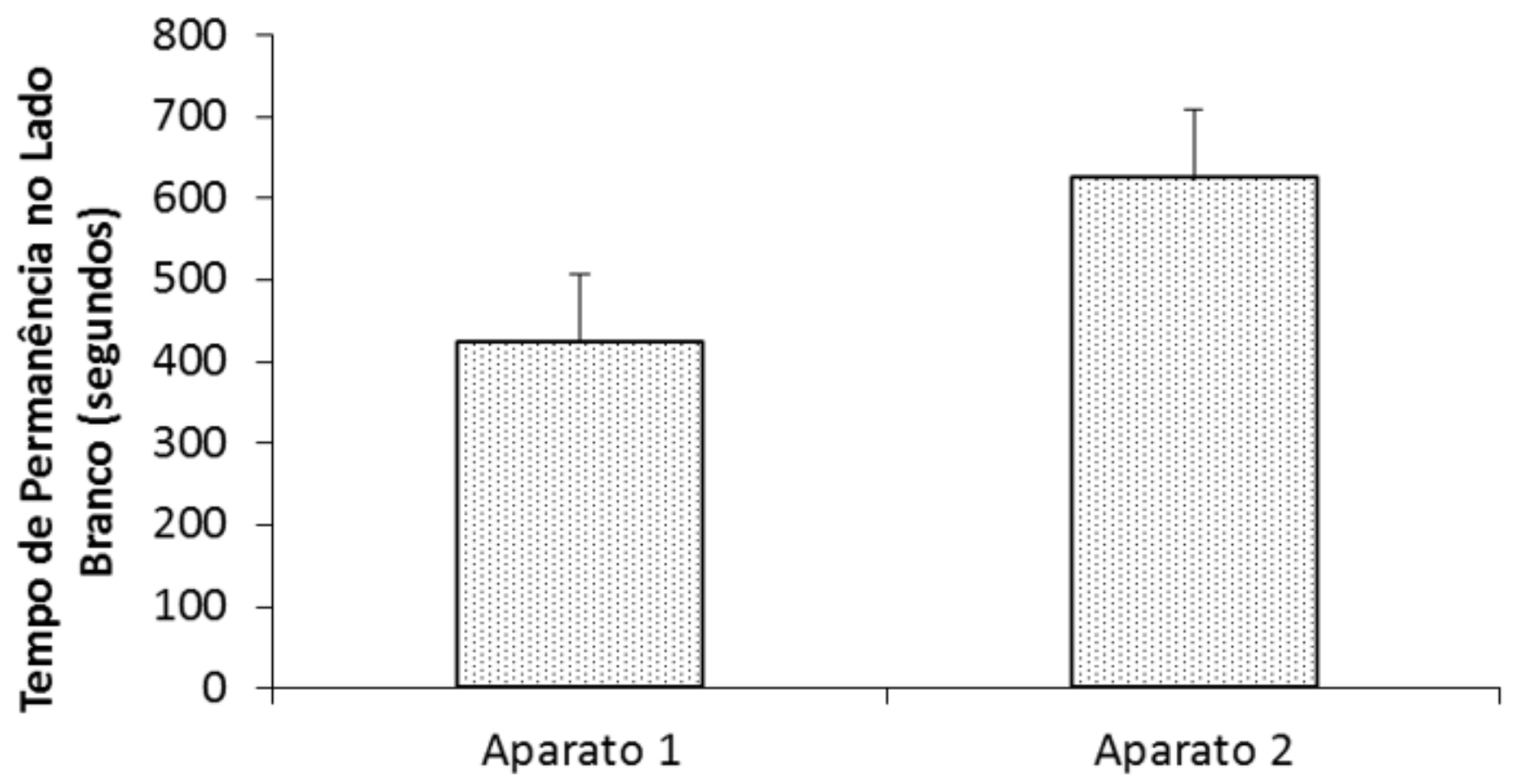

Aparatos de Isolamento

Figura 2 - Comparação do tempo de permanência do lado branco do aquário teste para ambos os aparatos, sem diferença significante $(\mathrm{F}(1,28)=0,000, \mathrm{p}=1,000)$.

\section{DISCUSSÃO}

Ciprinídeos foram colocados em uma área central de um aquário onde podiam escolher nadar para o ambiente preto ou para o ambiente branco. Fatores como a primeira escolha do ambiente, quantas vezes cada ambiente foi visitado e o tempo de permanência em cada ambiente foram considerados, chegando-se a conclusão que existe uma preferência pelo lado escuro (Gouveia Jr, Zampieri et al., 2005; Maximino, Brito et al., 2010).

\section{CONCLUSÃO}

A espessura do material utilizado para a fabricação dos aparatos, suas dimensões, o tipo de isolamento, os tipos e o posicionamento dos exaustores (coolers), e o posicionamento da iluminação não afetam os testes comportamentais que derivam da manutenção dos sujeitos dentro deles.

Ambos os aparatos demonstraram ser igualmente eficazes na manutenção do Zebrafish para testes comportamentais com variável luminosa.

\section{REFERÊNCIAS}

BILOTTA, J.; SASZIK, S. The zebrafish as a model visual system. Int. J. Devl Neuroscience, v. 19, p. 621-629, 2001.

BRITO, T. M. et al. Padrões de preferência Claro-escuro de duas espécies de teleósteos em um 
paradigma de teste-reteste. XXII Reunião Anual da Federação de Sociedades de Biologia Experimental (FeSBE) 2007.

DIAS, C. A. G. D. M. Preferência Claro/Escuro em Danio rerio: Efeitos do horário da Coleta e de Regime de Luz. 2010. 34 (Mestrado). Instituto de Ciências Biológicas, Universidade Federal do Pará

GOUVEIA JR, A. et al. Preference of goldfish (Carassius aurautus) for dark places. Revista de Etologia, v. 7, n. 2, p. 63-66, 2005.

HOUART, C. Zebrafish as an Experimental Organism. Encyclopedia of Life Sciences, p. 1 - 5, 2001.

MAXIMINO, C. et al. Scototaxis as anxiety-like behavior in fish. Nature Protocols, v. 5, p. 221-228, 2010.

MAXIMINO, C. et al. Scototaxis an anxiety-like behavior in fish. Nature Protocols, v. 4, n. 12, p. 1-8, 2009.

SERRA, E. L., MEDALHA, C.C., MATTIOLI, R. . Natural preference of zebrafish (Danio rerio) for a dark environment. Brazilian Journal of Medical and Biological Research v. 32, p. 1551-1553, 1999.

SPENCE, R. et al. The distribution and habitat preferences of the zebrafish in Bangladesh. Journal of Fish Biology, v. 69, p. 1435-1448, 2006.

SPENCE, R. et al. The behaviour and ecology of the zebrafish, Danio rerio. Biological Reviews, v. 83 p. 13-34, 2008.

${ }^{[1]}$ Biólogo. Doutor em Teoria e Pesquisa do Comportamento. Docente e Pesquisador do Instituto Federal do Amapá - IFAP.

${ }^{[2]}$ Biólogo. Especialista em Analises Clinicas. Docente e Pesquisador do Curso Anglo Bauru SP.

[3] Biomédica. Doutora em Doenças Tropicais. Docente e Pesquisadora da Universidade Federal do Amapá, AP. Pesquisador colaboradora do Núcleo de Medicina Tropical da UFPA (NMT-UFPA).

${ }^{[4]}$ Biólogo. Doutor em Medicina/Doenças Tropicais. Docente e Pesquisador na Universidade Federal do Pará - UFPA.

${ }^{[5]}$ Psicólogo. Doutor em Teoria e Pesquisa do Comportamento. Docente e Pesquisador na Universidade Federal do Pará - UFPA.

[6] Psicólogo. Mestre em Teoria e Pesquisa do Comportamento. Pesquisador colaborador na Universidade Federal do Pará - UFPA.

${ }^{[7]}$ Doutora em Psicanálise Clínica, Pesquisadora pelo Centro de Pesquisa e Estudos Avançados. 
${ }^{[8]}$ Psicólogo. Doutor em Neurociências e Comportamento. Livre Docente e Pesquisador na Universidade Federal do Pará - UFPA.

\section{PUBLIQUE SEU ARTIGO CIENTÍFICO EM:}

https://www.nucleodoconhecimento.com.br/enviar-artigo-cientifico-para-submissao 